\title{
Developing a Nicotiana benthamiana - Citrus tristeza virus model system to study superinfection exclusion
}

\author{
Mariana Suguieda, Sung-Hwan Kang, \& Svetlana Yuryevna Folimonova \\ University of Florida
}

Faculty mentor: Svetlana Yuryevna Folimonova, Department of Plant Pathology

\begin{abstract}
Citrus tristeza virus (CTV) is the causal agent of economically devasting diseases that affect global citrus production. CTV has demonstrated superinfection exclusion (SIE), a phenomenon where a preexisting viral infection excludes a secondary infection by the same or closely related virus. This phenomenon has been used to develop cross-protection measures against CTV but there have been erratic results with this approach. Understanding of SIE by CTV on the molecular level has been challenging, especially due to time-consuming trials of over a year when citrus plants are used in the experiments. The purpose of this study was to develop a model system to study SIE by CTV by using a more amenable species, Nicotiana benthamiana, rather than citrus plants. cDNA constructs of CTV containing extra genes of the green or red fluorescent proteins (GFP/RFP) were engineered and transformed into Agrobacterium tumefaciens to visualize virus movement and SIE within the $N$. benthamiana plants by using the agro-infiltration approach for the virus constructs delivery. The constructs carrying the full-length wild-type virus with the additional GFP- or RFP-encoding gene and the GFP-tagged hybrid constructs with deletion in the p33 gene were infiltrated with the agro-suspension and visualized under a microscope. The images captured were analyzed for peaks in grayscale values in the green or red channels along a line. The constructs used in the study behaved similarly in $N$. benthamiana and Citrus macrophylla but the trial time was significantly shortened with the $N$. benthamiana plants. Thus, $N$. benthamiana is a suitable alternative for studying SIE by CTV with the constructs in this study.

Keywords: superinfection exclusion, Citrus tristeza virus, Nicotiana benthamiana
\end{abstract}

\section{Introduction}

Citrus tristeza virus (CTV), of the family Closteroviridae, is the causal agent of two economically devastating diseases that dramatically affect citrus industries globally: quick decline and stem pitting (Bar-Joseph, Marcus, \& Lee., 1989; Moreno, Ambrós, Albiach-Marti, Guerri, \& Pena., 2008). Quick decline results in the death of the entire citrus plant, and stem pitting results in unmarketable fruit. CTV is vectored by aphids with its host range including citrus and citrus relatives that infect phloem-associated cells.

Protection against CTV has mainly focused on cross-protection, the introduction of a mild virus isolate as a protective measure against isolates causing severe disease (Folimonova, 2013). In the citrus industry, this approach against CTV had erratic results. When successful, it 
maintained good production of certain citrus varieties in some regions (Lee \& Keremane, 2013). However, protecting isolates were not always available in other regions or for different varieties, and their evaluation took several years (Folimonova, 2013). In many cases, mild CTV isolates failed to protect citrus or worked only for a short time.

At the molecular level, there has been little understanding of CTV cross-protection. One of the mechanisms that explains cross-protection is superinfection exclusion (SIE), which is an ability of an established virus infection to prevent secondary infection by the same or closely related virus (Author, 2012). Understanding SIE by CTV has been one of the main focuses of Dr. Folimonova's research group, and her group previously demonstrated that SIE by CTV is mediated by viral p33 protein and the 5'-proximal coding region of the virus (Folimonova, 2012).

Although citrus is the natural host of CTV, studying the mechanism of SIE in the model system based on citrus trees is challenging and time-consuming. Many plant viruses and their molecular mechanisms are studied in more amenable model systems using herbaceous species like Arabidopsis thaliana or Nicotiana benthamiana by expressing cDNA constructs cloned into a binary vector and transformed into Agrobacterium tumefaciens for the delivery into the plants by agro-infiltration (Ambrós et al., 2011).

The goal of this project was to develop a tobacco-CTV model system to study the mechanism of SIE, which adapts a citrus-CTV pathosystem and significantly shortens the overall term required for the study. To demonstrate and evaluate SIE in $N$. benthamiana, the green/red fluorescent protein (GFP/RFP) -tagged CTV constructs that were engineered based on a cDNA clone of CTV were monitored for virus distribution and movement in the inoculated plants. Using A. tumefaciens (agro) - mediated infiltration, plants were simultaneously inoculated with viral constructs known to exclude each other and viral constructs that would not exclude each other in citrus.

\section{Materials and Methods}

\section{Virus Constructs and Agroinfiltration}

All constructs used in this study were based on the full-length cDNA clone of CTV-T36. GFP-tagged CTV-T36 (LGFP), RFP-tagged CTV-T36 (LRFP), and GFP-tagged p33 deletion mutant CTV-T36 ( $\Delta$ p33) were constructed as described previously (Atallah et al., 2016; Author , 
2012; Author et al., 2015) (Figure 1). These constructs were transformed into A. tumefaciens by heat shock and selected from Luria-Bertani (LB) agar plates with appropriate antibiotics (50 $\mathrm{mg} / \mathrm{ml}$ rifampicin and $25 \mathrm{mg} / \mathrm{ml}$ kanamycin) as previously described (Ambrós et al., 2011; ElMohtar \& Dawson, 2014). For agroinfiltration, the transformed Agrobacterium cells were then inoculated in LB broth with the appropriate antibiotics, grown overnight, pelleted and then resuspended in a buffer containing $10 \mathrm{mM}$ 2-(N-morpholino) ethane sulfonic acid (MES, $\mathrm{pH}$ 5.85), $10 \mathrm{mM} \mathrm{MgCl}_{2}$ and $150 \mathrm{mM}$ acetosyringone at an Optical Density at 600nm (O.D.600nm) of 0.1 for LRFP and 0.2 for the LGFP and $\Delta \mathrm{p} 33$ as described in Kang et. al (2015). After two hours of shaking incubation at room temperature, the suspensions were infiltrated using a needleless syringe into the abaxial side of three-week old $N$. benthamiana plants.

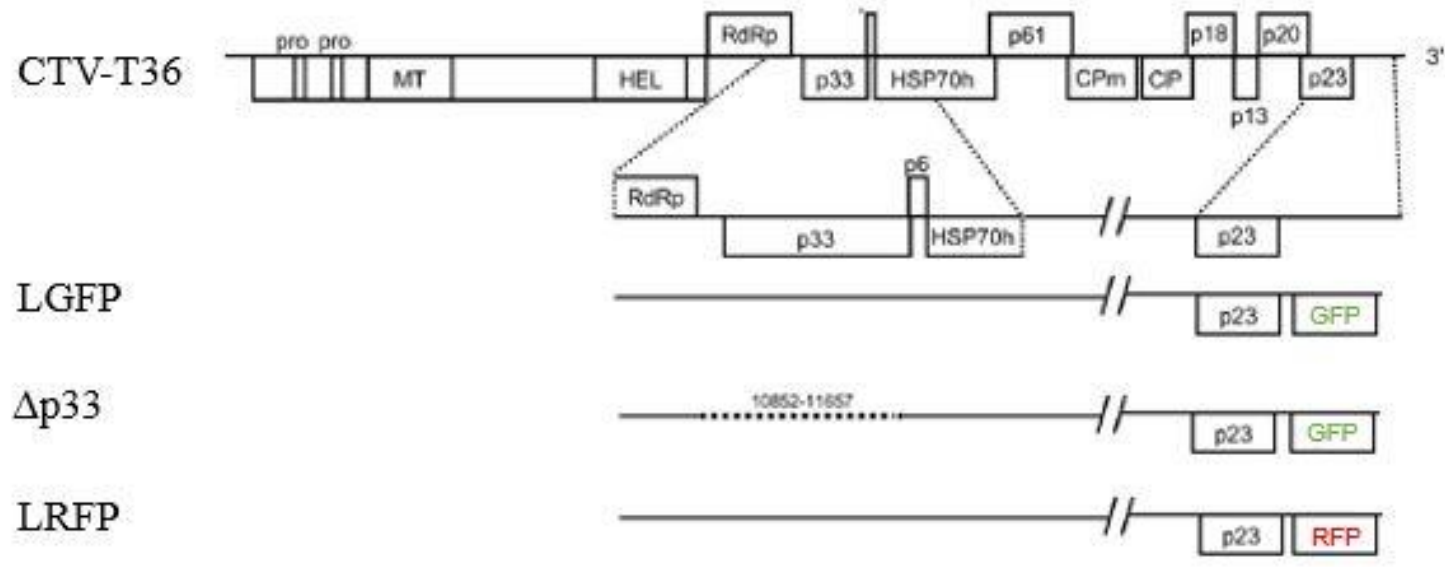

Figure 1. Schematic representation of the CTV-T36 genome and the constructs used in this study. The boxes represent open reading frames (ORF) and their products. Pro, papain-like protease domain; MT, methyltransferase; HEL, helicase; RdRp, RNA-dependent RNA polymerase; HSP70h, HSP70 homolog; CPm, minor coat protein; CP, coat protein. The derivatives are shown under the enlarged genome segment. LGFP and $\Delta \mathrm{p} 33$ have the GFP ORF inserted between the p23 gene and 3' NTR of the CTV genome under the native promoter of the CTV CP sgRNA Deletion in the $\mathrm{p} 33$ gene for the $\Delta \mathrm{p} 33$ construct is indicated by the dotted lines. LRFP has the RFP ORF inserted between the p23 gene and 3' NTR of the CTV genome under the native promoter of the CTV CP sgRNA. Adapted from Attalah (2016), Author (2012) and Author (2015).

\section{Serial Dilution and Co-infiltration Experiments}

Plants were infiltrated with the GFP-tagged constructs (LGFP and $\Delta \mathrm{p} 33$ ) and the RFP-tagged construct (LRFP) to determine the concentration of the suspension that would lead to a similar titer for the competing constructs, estimated by the number of fluorescing cells expressing green or red fluorescence, respectively, using a series of serial dilution experiments. First, plants were individually infiltrated with agro-suspensions of LGFP and LRFP with the following 
concentrations: O.D.600nm $=0.2,0.1$, and 0.05 . Then, plants were co-infiltrated with the same concentration and varying combinations of concentrations: O.D.600nm = LGFP 0.1 vs. LRFP 0.1, LGFP 0.2 vs. LRFP 0.1, LGFP 0.1 vs. LRFP 0.05, and LGFP 0.05 vs. LRFP 0.1 (Table 1).

Table 1. Serial dilution and co-infiltration concentrations

\begin{tabular}{ccc}
\hline $\begin{array}{c}\text { LGFP (single infiltration) } \\
\text { O.D.600nm }\end{array}$ & $\begin{array}{c}\text { LRFP (single infiltration) } \\
\text { O.D.600nm }\end{array}$ & $\begin{array}{c}\text { LGFP + LRFP (co-infiltration) } \\
\text { O.D.600nm }\end{array}$ \\
\hline 0.2 & 0.2 & 0.1 vs. 0.1 \\
0.1 & 0.1 & 0.2 vs. 0.1 \\
0.05 & 0.05 & 0.1 vs. 0.05 \\
& & 0.05 vs. 0.1 \\
\hline
\end{tabular}

For the co-infiltration experiments, 15 plants were infiltrated with a combination of agrosuspensions along with a set of 5 plants each infiltrated with an agro-suspension harboring a single virus construct. Two leaves were infiltrated per plant. These trials were repeated three times.

\section{Fluorescence Microscopy and Image Analysis}

Seven to ten days post-infiltration (dpi), the infiltrated leaves were examined for red and green fluorescence with a Leica MZ10F fluorescence dissecting stereomicroscope with an attached Leica DF310FX camera (Leica, Wetzlar, Germany). Images were captured using the LAS X 3.0.16120.2 software (Leica, Wetzlar, Germany). This examination was repeated about three weeks post-infiltration (wpi) with the upper, un-infiltrated leaves of the plants that were demonstrating systemic infections.

Images captured with the Leica MZ10F microscope were analyzed using ImageJ 1.52n software (NIH, USA). The images were measured for mean grayscale pixels on the split red and green channels of the serial dilution experiment images and the plot profiles of the grayscale red and green channels were measured and plotted against each other for the co-infection experiments.

\section{Reverse Transcription- Polymerase Chain Reaction (RT-PCR)}

Tissue samples (three leaf discs per treatment) of the coinfection experiments were taken and stored at -80 degrees Celsius. The samples were ground with liquid nitrogen and total RNA was 
extracted using the Direct-zol RNA extraction kit according to the manufacturer (Zymo Research, Irvine, California). RNA extracts were resuspended in $40 \mu \mathrm{L}$ of RNase-free water and the concentration was measured with a NanoDrop-UV-Vis Spectrophotometer (Thermo Fisher Scientific, Wilmington, DE, USA). The RNA extracts were then diluted with the RNase-free water to $10 \mathrm{ng} / \mathrm{mL}$ and stored at -80 degrees Celsius. The Open Reading Frames (ORFs) of GFP and RFP were amplified by RT-PCR using the primers in Table 2. The PCR products were then run on a $1 \%$ agarose gel to confirm infection along with a negative control using DNase and RNase free water.

Table 2. List of primers used for detection of viruses

\begin{tabular}{|c|c|c|c|c|}
\hline $\begin{array}{l}\text { Primer } \\
\text { Set }\end{array}$ & Sequence (5' to 3') & $\begin{array}{l}\text { Product size } \\
\text { (bp) }\end{array}$ & $\begin{array}{l}\text { Position in the CTV genome } \\
\text { (nt) }\end{array}$ & Refs. \\
\hline GFP & $\begin{array}{l}\text { (+) ctggagttgtcccaattcttg } \\
(-) \text { cgaaagggcagattgtgtcga }\end{array}$ & 599 & $\begin{array}{c}\text { Sequences within the GFP } \\
\text { ORF }\end{array}$ & $\begin{array}{l}\text { Atallah et al. } \\
\quad(2016)\end{array}$ \\
\hline RFP & $\begin{array}{c}(+) \text { aggagaacatgcacatgaagc } \\
(-) \text { gtcggcctccttgattctttc }\end{array}$ & 602 & $\begin{array}{c}\text { Sequences within the RFP } \\
\text { ORF }\end{array}$ & $\begin{array}{l}\text { Atallah et al. } \\
\qquad(2016)\end{array}$ \\
\hline
\end{tabular}

Note. Adapted from "A 5'-proximal region of the Citrus tristeza virus genome encoding two leader proteases is involved in virus superinfection exclusion," O. O. Attalah et al., 2016, Virology, 489, 111.

\section{Results \\ The Adequate Concentration of Agrobacterium Suspension for the Infiltration was Empirically Determined}

When the LGFP agro-suspension was infiltrated at O.D.600nm $=0.2$ along with the LRFP agrosuspension at O.D.600nm $=0.1$, the mean grayscale $(\mathrm{MG})$ value of the green and red color channels on ImageJ were 5.03 and 7.08 respectively (Figure 2A). This combination led to the closest MG values between the red and green channels when compared to the other concentration combination, which suggests a similar number of cells were infected by LGFP and LRFP. Combinations of LRFP at O.D.600nm= 0.05 and LGFP at O.D.600nm $=0.1$ led to a weaker red fluorescence under the microscope (Figure 2B). When infiltrated at the same concentration at O.D.600nm $=0.1$, red fluorescence from LRFP was much stronger than the green fluorescence from LGFP (Figure 2C). Combinations of LRFP at O.D.600nm= 0.1 and LGFP at O.D.600nm=0.05 led to a very strong red fluorescence that overwhelmed the green fluorescence (Figure 2D). Using ImageJ, the mean grayscale (MG) value in the green or red channel was used to determine which 
combination resulted in the closest viral titer between the competing viruses: GFP-tagged constructs at O.D.600nm $=0.2$ and LRFP at O.D.600nm $=0.1$.

A)

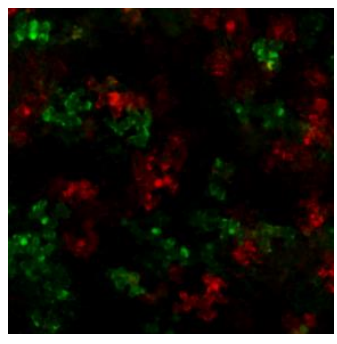

LGFP O.D.600nm = 0.2; $\mathrm{MG}=5.03$

LRFP O.D.600nm $=0.1$; $\mathrm{MG}=7.08$
B)

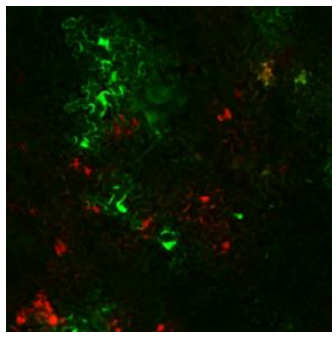

LGFP O.D.600nm = 0.1; $\mathrm{MG}=12.7$

LRFP O.D.600nm $=0.05$; $\mathrm{MG}=6.2$
C)

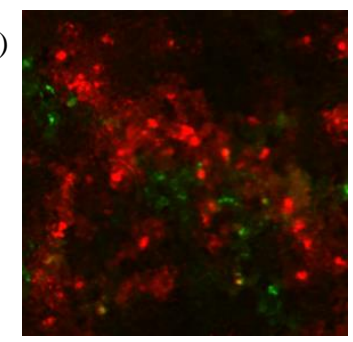

LGFP O.D.600nm = 0.1; $\mathrm{MG}=13.9$

LRFP O.D.600nm = 0.1; $\mathrm{MG}=43.4$
D)

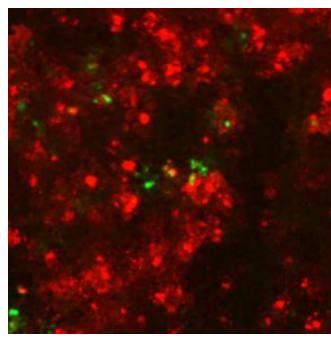

LGFP O.D.600nm $=0.05$; $\mathrm{MG}=13.7$

LRFP O.D.600nm = 0.1; $\mathrm{MG}=64.1$

Figure 2. Expression of the competing viral constructs by the Leica microscope with their O.D.600nm concentrations and mean grayscale (MG) value that measures the average gray pixels in the green and red channels. (A) The combination of concentrations for LGFP and LRFP used in the study given that the MG of the green and red channels were similar. (B) LGFP signal was too strong compared to the LRFP signal. (C) The LRFP signal overwhelmed the LGFP signal because LRFP tends to perform better against LGFP. (D) The LRFP signal was too strong in this combination.

\section{Upon Co-Infiltration, LGFP and LRFP Excluded Each Other in N. benthamiana}

Following the serial dilution results, several sets of $N$. benthamiana plants were co-infiltrated with agrobacterium-suspensions of LGFP + LRFP. The plot profiles of the images' grayscale green and red channels varied greatly when plotted against each other (Figure 3). The merged images of LGFP + LRFP in the infiltrated or systemically infected leaves did not show many coinfected cells, which would appear orange or yellow (Figure 3A and 3B). To quantitatively validate this, the grayscale green and red channels were plotted against each other to measure the grayscale levels along a line. These plot profiles of LGFP + LRFP showed varied peaks in different locations meaning there was no overlap in grayscale values that would indicate a coinfected cell (Figure 3C and 3B). Thus, LGFP +LRFP succesfully excluded each other at the cellular level in both infiltrated and systemically infected leaves.

Infiltrated Leaf of LGFP + LRFP

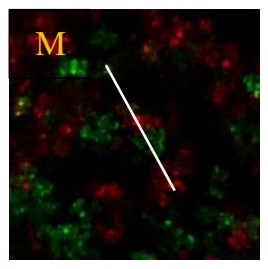

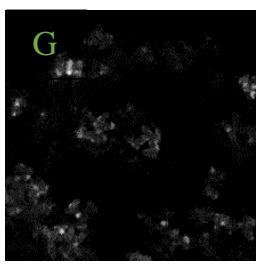

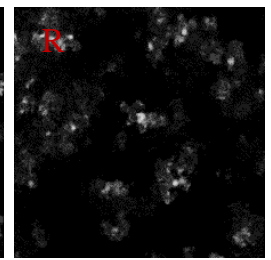

B) Systemic Leaf Infection of LGFP + LRFP

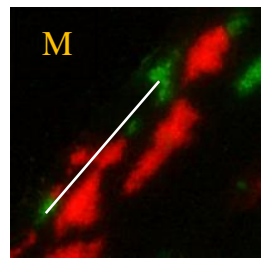

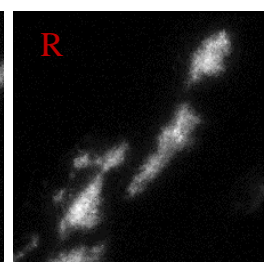


C) Grayscale Green/Red Plot Profiles of Infiltrated Leaves

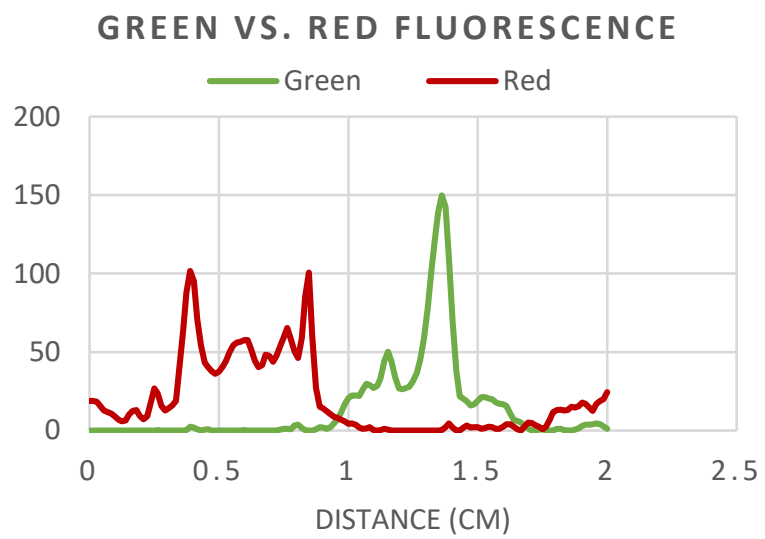

D) Grayscale Green/Red Plot Profiles of Systemic Leaf

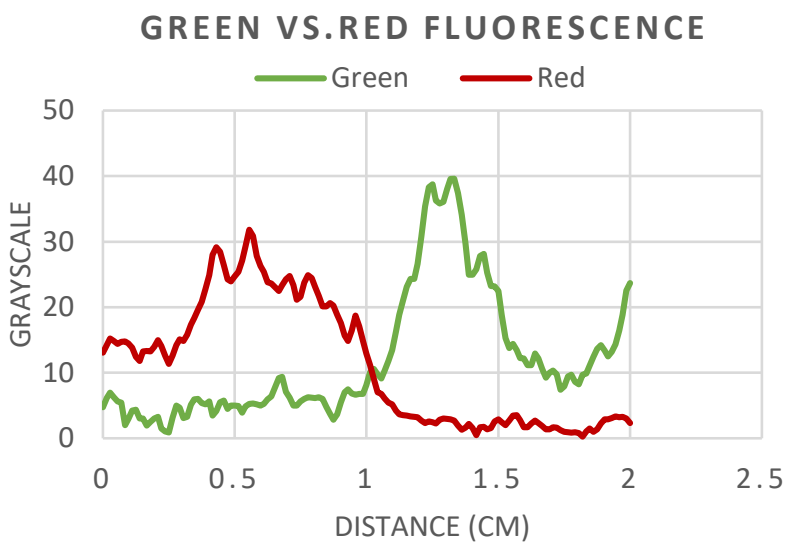

Figure 3. (A) Merged LGFP + LRFP co-infiltration of infiltrated leaf images (10dpi) alongside grayscale green and red channels. (B) Merged images of LGFP + LRFP co-infiltration in systemic leaf infection (30 dpi) alongside grayscale green and red channels and line used in plot profiles. (C) Plot profile and measurement of fluorescing pixels on each (green or red) channel corresponding with line in the merged infiltrated leaf image above. (D) Plot profile and measurement of fluorescing pixels on each (green or red) channel corresponding with line in the merged systemically infected leaf image above. The tables show varied peaks in the green and red grayscale profiles indicating no co-infected cells. Plot line unit $(\mathrm{cm})$ corresponds to the image dimensions not the cell size. M for merged green and red channels, G for Grayscale Green Channel, and R for Grayscale Red Channel. A white line indicates the plot used for quantification of fluorescing pixels.

\section{Upon Co-Infiltration, $\Delta$ p33 and LRFP Did Not Exclude Each Other in N. benthamiana}

Next, several sets of $N$. benthamiana plants were co-infiltrated with agrobacteriumsuspensions of $\Delta$ p33 + LRFP. The merged green and red channel images of infiltrated and systemically infected leaves showed some cells being orange or yellow, indicating co-infected cells (Figure 4A and 4B). Analysis by ImageJ of the grayscale green and red channels of infiltrated and systemically infected leaves showed plot profiles that had similar peaks and direction between the two lines (Figure 4C and 4D). Co-infiltration of $\Delta \mathrm{p} 33+$ LRFP led to several co-infected cells in both infiltrated and systemically infected leaves, meaning they did not exclude each other at the cellular level.

A) Infiltrated Leaf of $\Delta$ p33 + LRFP
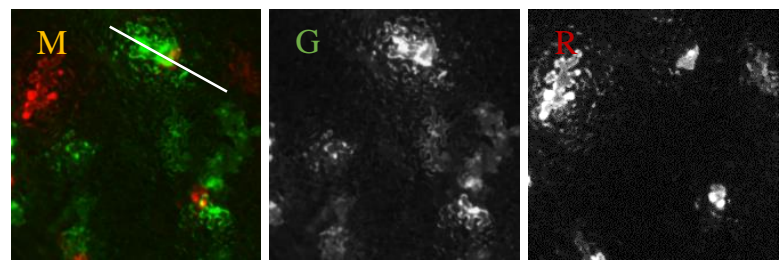

C) Grayscale Green/Red Plot Profiles of Infiltrated Leaves
B) Systemic Leaf Infection of $\Delta$ p33 + LRFP
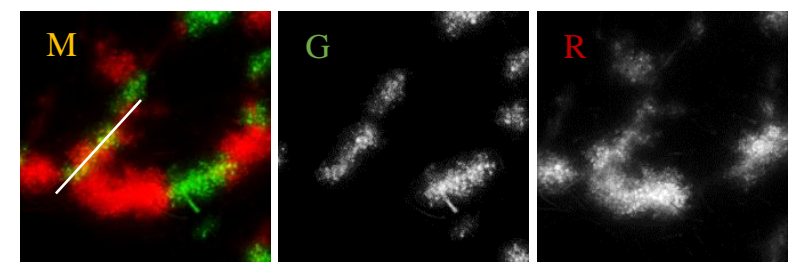

D) Grayscale Green/Red Plot Profiles of Systemic Leaf 

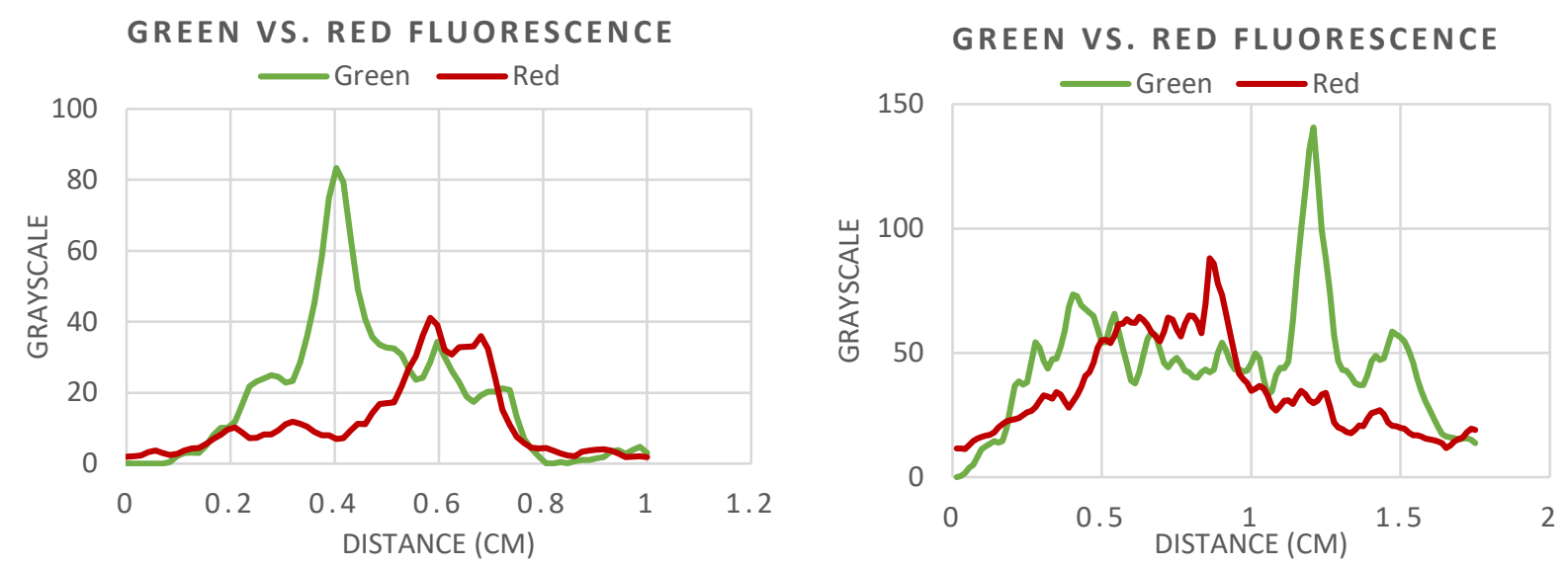

Figure 4. (A) Merged $\Delta \mathrm{p} 33+$ LRFP co-infiltration of infiltrated leaf images (10dpi) alongside grayscale green and red channels and line used in the plot profiles. (B) Merged images of $\Delta$ p33 + LRFP co-infiltration in systemic leaf infection (30 dpi) alongside grayscale green and red channels and line used in plot profiles. (C) Plot profile and measurement of fluorescing pixels on each (green or red) channel corresponding with line in the merged infiltrated leaf image above. The table shows closely mirrored lines between $0.56 \mathrm{~cm}$ and $0.75 \mathrm{~cm}$ that suggests a co-infected cell along the line measured. (D) Plot profile and measurement of fluorescing pixels on each (green or red) channel corresponding with line in the merged systemically infected leaf image above. The table shows closely mirrored lines between $0.5 \mathrm{~cm}$ and $0.1 \mathrm{~cm}$ that suggests a co-infected cell along the plot line measured. Plot line unit $(\mathrm{cm})$ corresponds to the image dimensions not the cell size. $\mathrm{M}$ for merged green and red channels, $\mathrm{G}$ for Grayscale Green Channel, and R for Grayscale Red Channel. A white line indicates the plot used for quantification of fluorescing pixels.

\section{The Presence of CTV Viruses in the Infiltrated Leaves was confirmed by RT-PCR}

Two samples of each treatment (LGFP/LRFP/LGFP+LRFP/ $\Delta \mathrm{p} 33 / \Delta \mathrm{p} 33+\mathrm{LRFP})$ from infiltrated and systemically infected leaves, with three leaf discs per sample, were collected during the study to confirm the presence of each virus in singly-infiltrated and co-infiltrated plants by RT-PCR. RT-PCR products were confirmed by gel electrophoresis. Co-infiltrated treatments of LGFP + LRFP from infiltrated leaves were confirmed by both samples collected (Figure 5A). In the systemically infected leaf samples of LGFP + LRFP, all samples were confirmed for GFP and RFP ORF amplification. However, of the two systemically infected LGFP + LRFP samples collected, one sample did not amplify GFP (Figure 5B). Infiltrated and systemically infected leaves with $\triangle \mathrm{p} 33$ + LRFP successfully amplified the GFP and RFP ORFs (Figure 5C). No samples were collected from systemically infected leaves treated with $\Delta \mathrm{p} 33$ only. 

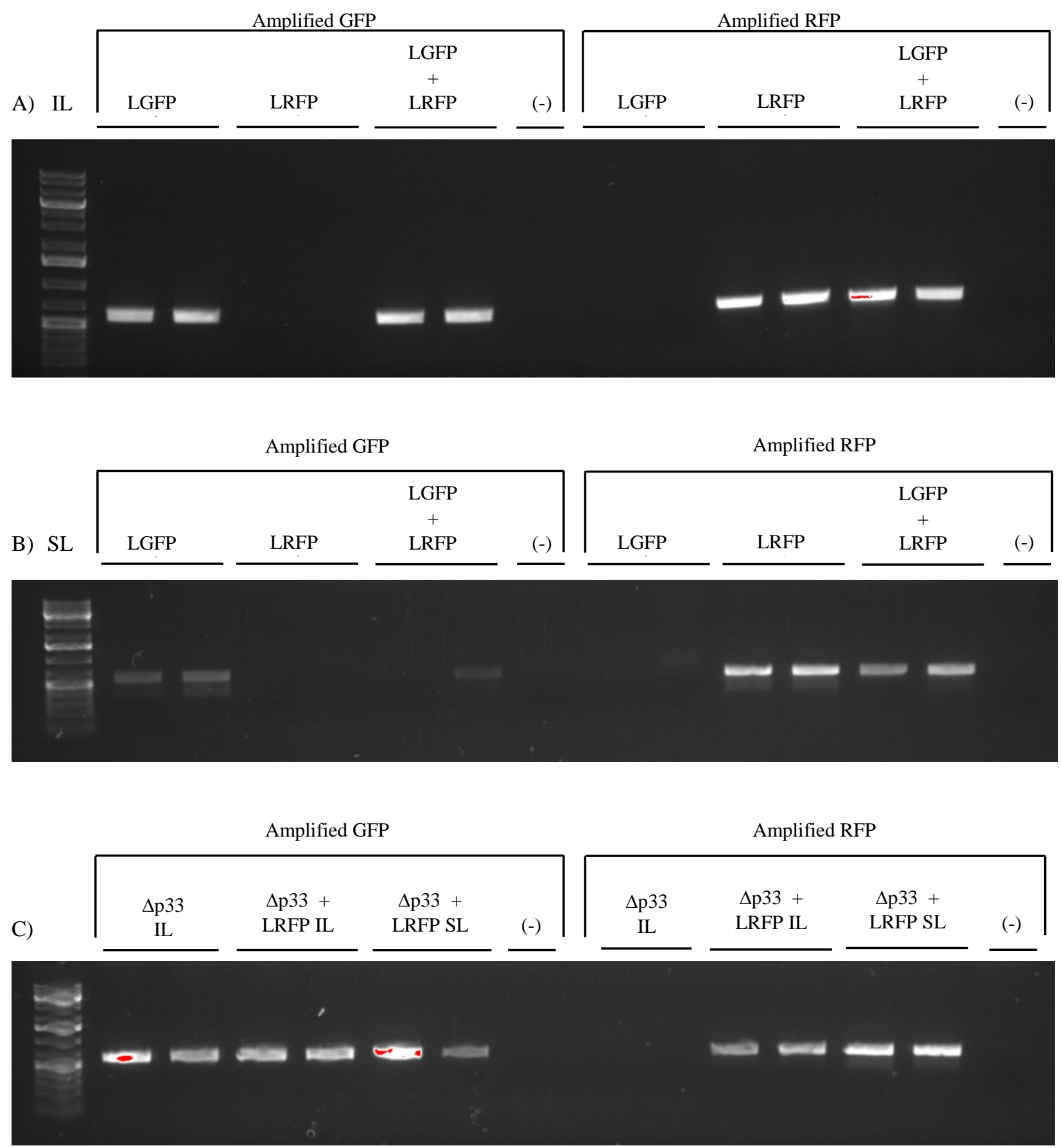

Figure 5. Gel electrophoresis of RT-PCR products amplifying the ORFs of GFP and RFP of virus constructs from total RNA extracted from the infiltrated N. benthamiana plants. (A) Infiltrated leaf samples with correct amplification and band visualization based on GFP or RFP primers used in the RT-PCR. (B) Systemic leaf samples with correct amplification and band visualization on GFP or RFP primers used apart from the first sample in the LGFP + LRFP lane that did not amplify GFP. However, the second sample of that set did successfully amplify GFP. (C) Correct amplification and visualization of GFP or RFP in infiltrated leaves and systemic infection other than the $\Delta$ p33 systemic infection. IL for Infiltrated Leaves and SL for Systemic Leaves. 


\section{Discussion}

In previous studies, Citrus macrophylla trees were infected with LGFP and LRFP sequentially, meaning the plant was inoculated with one virus first and left for the infection to be established for months and then challenged with the second virus (Author, 2012). This timeframe was a difficulty when developing this study because $N$. benthamiana leaves are not viable long enough for a sequential infiltration. Therefore, the leaves were co-infiltrated with an agrosuspension of a GFP-tagged construct and LRFP. The serial dilution experiments were meant to determine the O.D. concentrations of the agro-suspensions that would result in an even and equal spread of infected cells throughout the leaf.

Despite these changes, LGFP and LRFP did not co-infect the same cells in either infiltrated or systemically infected leaves, so they successfully excluded each other at the cellular level in $N$. benthamiana plants. This result is consistent with studies that show SIE by CTV in $C$. macrophylla (Folimonova, Robertson, Shilts, Folimonov, Hilf, Garnsey, \& Dawson, 2009). Likewise, $\Delta$ p33 and LRFP did co-infect the same cells, unable to exclude each other, similar to studies of SIE by CTV in C. macrophylla with the same constructs (Folimonova, 2012). The overall aim of this study was to develop a system to study SIE by CTV in a shorter time. With $N$. benthamiana, the study time is approximately 7 to $10 \mathrm{dpi}$ in the infiltrated leaves and around $30 \mathrm{dpi}$ for the systemically infected leaves compared to the study period of over a year in citrus plants. This is a significant shortage in time that will allow researchers to better understand the mechanisms of SIE.

In the future, other hybrid clones of CTV should be tested for further validation that $N$. benthamiana system demonstrated in this study is a suitable for studying SIE by CTV. These hybrid clones can include T68 p33 or T68L1L2, a construct where the p33 gene or L1L2 genomic region of the CTV-T36 strain has been replaced with the p33 gene or L1L2 genomic region of CTV-T68 strain, of which SIE had been tested in C. macrophylla system (Atallah et al., 2016). Additionally, there are reverse combinations such as the RFP-tagged $\Delta \mathrm{p} 33$ (rather than GFP tagged) with LGFP that need to be evaluated. So far, LGFP + LRFP and $\Delta \mathrm{p} 33+$ LRFP have demonstrated similar exclusion behaviors in $N$. benthamiana and C. macrophylla, making $N$. benthamiana a suitable alternative to citrus plants for the study of SIE by LGFP, LRFP and $\Delta \mathrm{p} 33$. 


\section{Acknowledgements}

I would like to thank Dr. Kang for hours of discussion and mentoring during this study. Additionally, I thank Dr. Folimonova for the support and opportunity she has given me. This project was funded by the University Scholars Program at the University of Florida.

\section{References}

Ambrós, S., El-Mohtar, C., Ruiz-Ruiz, S., Peña, L., Guerri, J., Dawson, W. O., \& Moreno, P. (2011). Agroinoculation of Citrus tristeza virus causes systemic infection and symptoms in the presumed nonhost Nicotiana benthamiana. Molecular Plant-Microbe Interactions, 24(10), 1119-1131. doi:10.1094/mpmi-05-11-0110

Atallah, O. O., Kang, S., El-Mohtar, C. A., Shilts, T., Bergua, M., \& Folimonova, S. (2016). A 5'proximal region of the Citrus tristeza virus genome encoding two leader proteases is involved in virus superinfection exclusion. Virology, 489, 108-115. doi:10.1016/j.virol.2015.12.008

Bar-Joseph, M., Marcus, R. \& Lee, R. F. (1989). The continuous challenge of Citrus tristeza virus control. Annual Review of Phytopathology, 27, 291-316.

Bergua, M., Kang, S., \& Folimonova, S. Y. (2016). Understanding superinfection exclusion by complex populations of Citrus tristeza virus. Virology, 499, 331-339. doi:10.1016/j.virol.2016.10.001

El-Mohtar, C., \& Dawson, W. O. (2014). Exploring the limits of vector construction based on Citrus tristeza virus. Virology, 448, 274-283. doi:10.1016/j.virol.2013.10.017

Folimonova, S. Y., Robertson, C. J., Shilts, T., Folimonov, A. S., Hilf, M. E., Garnsey, S. M., \& Dawson, W. O. (2009). Infection with strains of Citrus tristeza virus does not exclude superinfection by other strains of the virus. Journal of Virology, 84(3), 1314-1325. doi:10.1128/jvi.02075-09

Folimonova, S. Y. (2012). Superinfection exclusion is an active virus-controlled function that requires a specific viral protein. Journal of Virology, 86(10), 5554-5561. doi:10.1128/jvi.00310-12

Folimonova, S. Y. (2013). Developing an understanding of cross-protection by Citrus tristeza virus. Frontiers in Microbiology, 4(76). doi: 10.3389/fmicb.2013.00076

Kang, S., Bak, A., Kim, O., \& Folimonova, S. Y. (2015). Membrane association of a nonconserved viral protein confers virus ability to extend its host range. Virology, 482, 208-217. doi:10.1016/j.virol.2015.03.047

Lee, R. F., \& Keremane, M. L. (2013). Mild strain cross protection of tristeza: A review of research to protect against decline on sour orange in Florida. Frontiers in Microbiology, 4(259). doi: 10.3389.fmicb.2013.00259

Moreno, P., Ambrós, S., Albiach-Marti, M. R., Guerri, J. \& Pena, L. (2008), Citrus tristeza virus: A pathogen that changed the course of the citrus industry. Molecular Plant Pathology, 9, 251-268. doi:10.1111/j.1364-3703.2007.00455.x

Schindelin, J., Arganda-Carreras, I. \& Frise, E. (2012). Fiji: An open-source platform for biologicalimage analysis. Nature Methods, 9(7), 676-682. doi: 10.1038/nmeth.2019 